Case Report

\title{
A Case of a Young Patient with Acute Endocarditis and Challenging Diagnostic and Treatment Decisions
}

\author{
1,2Pau Rello, ${ }^{1,2}$ Teresa González-Alujas, ${ }^{2,3}$ Laura Escolà-Vergé, ${ }^{4,5}$ Albert Roque, ${ }^{2,6}$ Carlos Sureda, \\ ${ }^{1,2}$ Toni Soriano-Colomé, ${ }^{1,2}$ Gerard Oristrell, ${ }^{1,2}$ Antonia Sambola and ${ }^{1,2,7}$ Ignacio Ferreira-González \\ ${ }^{1}$ Department of Cardiology, Hospital Universitari Vall d'Hebron, Barcelona, Spain \\ ${ }^{2}$ Universitat Autònoma de Barcelona, Barcelona, Spain \\ ${ }^{3}$ Department of Infectious Diseases, Hospital Universitari Vall d'Hebron, Barcelona, Spain \\ ${ }^{4}$ Department of Radiology, Hospital Universitari Vall d'Hebron, Barcelona, Spain \\ ${ }^{5}$ Institut de Diagnòstic per la Imatge (IDI), Barcelona, Spain \\ ${ }^{6}$ Department of Cardiac Surgery, Hospital Universitari Vall d'Hebron, Barcelona, Spain \\ ${ }^{7}$ CIBER de Epidemiología y Salud Pública (CIBERESP), Madrid, Spain
}

\author{
Article history \\ Received: 18-03-2021 \\ Revised: 20-05-2021 \\ Accepted: 21-05-2021 \\ Corresponding Author: \\ Pau Rello \\ Department of Cardiology, \\ Hospital Universitari Vall \\ d'Hebron, Barcelona, Spain \\ and \\ Universitat Autònoma de \\ Barcelona, Barcelona, Spain \\ Email: prello@vhebron.net
}

\begin{abstract}
Despite advances achieved in recent years, Infective Endocarditis (IE) remains a disease associated with high mortality and morbidity. When it involves multiple locations at the same time, deciding the best treatment can become challenging. In some cases, especially in patients with prosthetic valve endocarditis, a definitive diagnosis can be difficult to achieve and multimodality imaging including Positron Emission Tomography/Computed Tomography Angiography (PET/CTA) has demonstrated improvement in the diagnostic yield. We present a case of a young patient with two previous thoracic surgeries who was admitted due to a severe Staphylococcus aureus IE affecting the mitral valve and presenting a questionable image in an aortic arch graft. This case illustrates the importance of the Endocarditis Team when it comes to difficult decisions regarding diagnosis and management in a disease with poor scientific evidence.
\end{abstract}

Keywords: Infective Endocarditis, Prosthetic Endocarditis, Cardiac 18FFDG-PET/CTA

\section{Introduction}

Despite advances achieved in recent years, Infective Endocarditis (IE) remains a disease associated with high mortality and morbidity (Habib et al., 2015). When it involves multiple locations at the same time, deciding the best treatment can become challenging. We present a case of a young patient with IE with difficult diagnostic and therapeutic decisions.

\section{Case}

We present a 22-year-old male patient with a past medical history of asthma. He had been treated for post ductal aortic coarctation on two occasions. The first surgery, at the age of 10 , consisted in coarctation repair with resection and end-to-end anastomosis through a left posterolateral thoracotomy. The second one, due to recoarctation at the age of 19 , required a medium sternotomy and consisted in enlarging the effective aortic area using a Dacron graft. He had a bicuspid aortic valve and a recent Transthoracic Echocardiogram (TTE) showed a mild aortic stenosis and regurgitation with no other valve disease.

He was admitted to a non-tertiary hospital due to fever spikes, hypogastric pain, nausea and vomiting. The physical examination revealed an apical systolic murmur. Initial blood test showed leukocytosis, neutrophilia and elevation of inflammatory parameters. Blood cultures were drawn and empiric antibiotic therapy with cefotaxime and vancomycin was started. The blood cultures were all positive for Methicillin-susceptible Staphylococcus aureus (MSSA) so the antibiotic treatment was changed to cefazolin $2 \mathrm{~g}$ every $8 \mathrm{~h}$. A TTE and Transesophageal Echocardiogram (TEE) were performed, showing a very large and mobile vegetation (maximum diameter of $22 \mathrm{~mm}$ ) attached to the A3 segment of the mitral valve, associated with leaflet perforation and moderate mitral regurgitation. Severe aortic regurgitation without any evidence of infection and a thickening of the aortic arch graft without associated vegetations were also seen. An abdominal CT scan showed no evidence of embolic lesions. The patient was then transferred to the acute cardiac care unit of our center to decide the best treatment. 
At arrival, the patient was febrile without any symptoms or signs of heart failure. A TEE was repeated showing similar findings except for the presence of a mobile image in the aortic arch graft that could not differentiate between postsurgical changes and an infective vegetation. Therefore, an 18F-Fluorodeoxyglucose Positron Emission Tomography/Computed Tomography Angiography (18F-FDG-PET/CTA) was performed. PET/CTA showed intense focal FDG uptake at the mitral valve associated with a large vegetation, confirming native mitral valve endocarditis and ruling out infection at the aortic valve. Concerning the aortic arch graft, PET/CTA depicted mild FDG uptake at the graft associated with mild mural thickening and little calcifications, but without periaortic soft tissue reaction or fluid collections. These findings were not conclusive of infection of the aortic graft. The Endocarditis Team decided that the patient required urgent surgery with no further delay due to the high embolic risk and uncontrolled cardiac infection. Due to the lack of evidence of infection and the extreme risk of the intervention, it was decided not to replace the aortic arch graft. Surgery confirmed mitral valve IE complicated by paravalvular abscess and degenerative changes on the aortic bicuspid valve with no evidence of infection. Mitral and aortic valve replacement were performed. The aortic arch was not accessible to be evaluated during the surgery.

The patient progressed satisfactorily during the first days after surgery. Mitral valve cultures were positive and the aortic valve was sterile. However, 5 days after surgery the patient developed fever spikes again and blood cultures were positive again for MSSA. TEE was repeated, with no evidence of endocarditis and no changes in the image of the aortic arch patch. Since the patient continued to be febrile with positive blood cultures another TEE was performed, showing a significant size increase of the mobile image of the aortic arch graft and a new onset moderate periprosthetic mitral regurgitation (Fig. 1 and 2). A second PET/CTA scan was performed, which showed a periprosthetic mitral fistula, no evidence of infection of the aortic prosthetic valve and no significant changes at the aortic arch graft findings compared with the first scan (Fig. 3). Daptomycin (10 mg/kg/day) was added to cefazolin with negative blood cultures and the patient was discussed again by the Endocarditis Team. Since the findings were not conclusive of infection and the surgical risk of a reintervention was very high, it was decided to maintain antibiotic treatment.

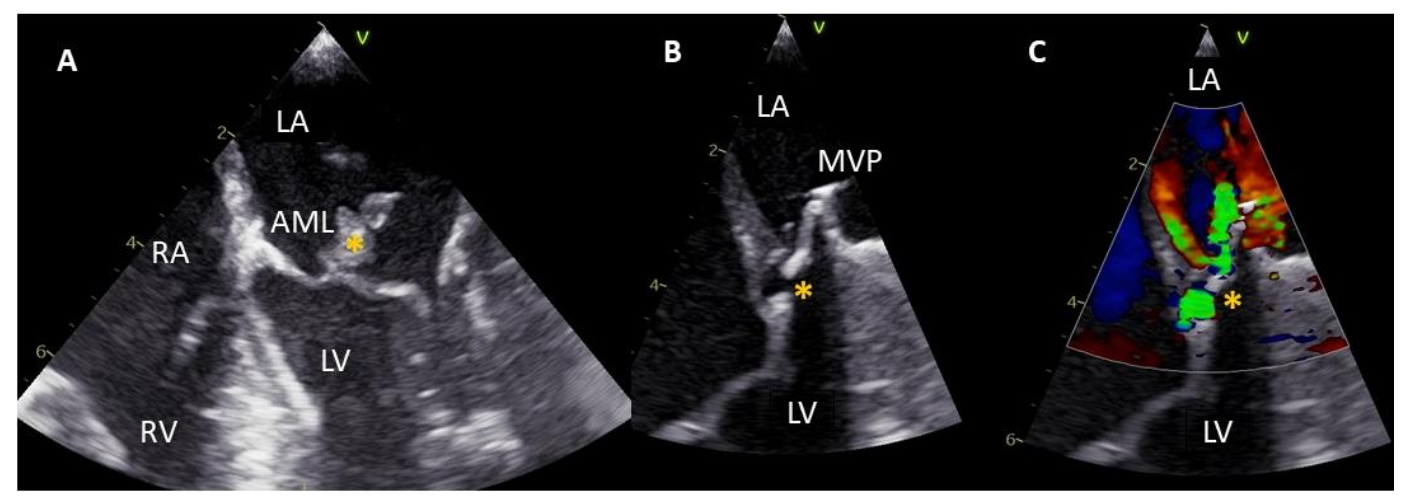

Fig. 1: Transoesophageal Echocardiograms. Mitral valve before and after surgery; (A) Transoesophageal echocardiogram at initial diagnosis. Mitral valve large and mobile vegetation (asterisk); (B, C) Transoesophageal echocardiogram after surgery with and without colour revealing an inferoseptal mitral periprosthetic dehiscence with moderate mitral regurgitation (asterisk); AML: Anterior Mitral Leaflet; LA: Left Atrium; LV: Left Ventricle; MVP: Mitral Valve Prosthesis; RA: Right Atria; RV: Right Ventricle
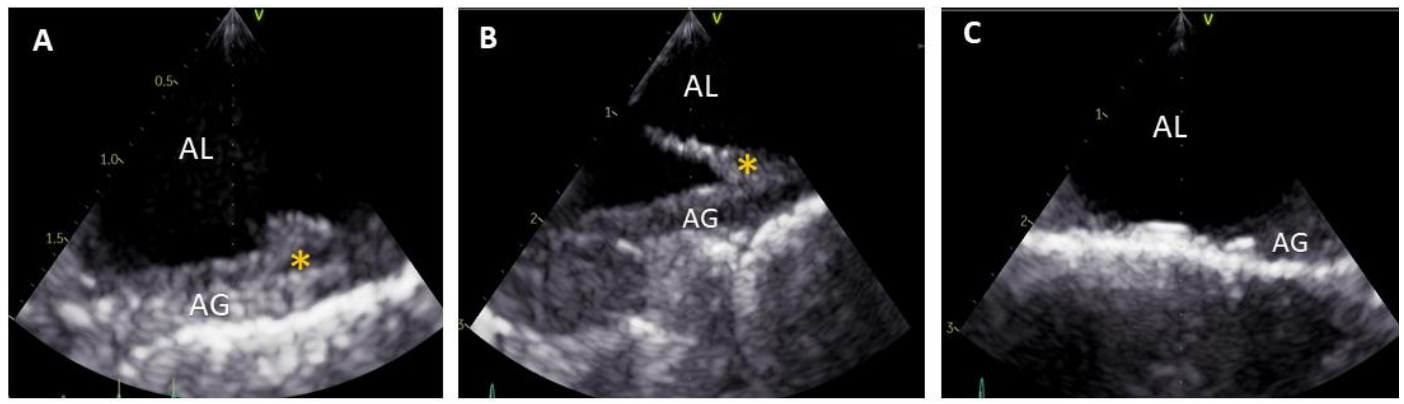

Fig. 2: Transoesophageal Echocardiograms of the aortic arch graft; (A) Transoesophageal echocardiogram at initial diagnosis. Mitral valve large and mobile vegetation (asterisk); (B, C) Transoesophageal echocardiogram after surgery with and without colour revealing an inferoseptal mitral periprosthetic dehiscence with moderate mitral regurgitation (asterisk); AML: Anterior Mitral Leaflet; LA: Left Atrium; LV: Left Ventricle; MVP: Mitral Valve Prosthesis; RA: Right Atria; RV: Right Ventricle 


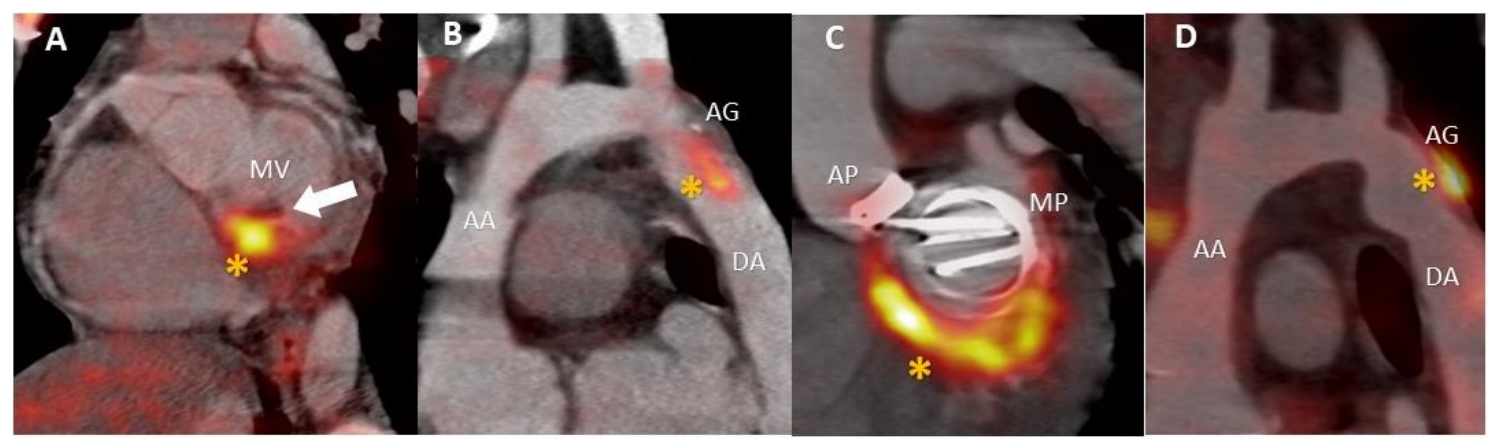

Fig. 3: 18F-FDG-PET/CTA at initial presentation and after cardiac surgery; (A) Cardiac PET/CTA before surgery showing intense mitral valve FDG uptake (asterisk) associated with a large vegetation (arrow). (B) PET/CTA also depicts FGD uptake in the aortic arch graft (asterisk) without perigraft inflammatory signs. (C) PET/CTA after surgery shows intense focal posterior periprosthetic mitral FDG uptake (asterisk). (D) Similar FGD uptake is seen in the aortic arch graft (asterisk), without relevant changes compared with the initial test; AA: Ascending Aorta; AG: Aortic Graft; AP: Aortic Prosthesis; DA: Descending Aorta; MP: Mitral Prosthesis; MV: Mitral Valve

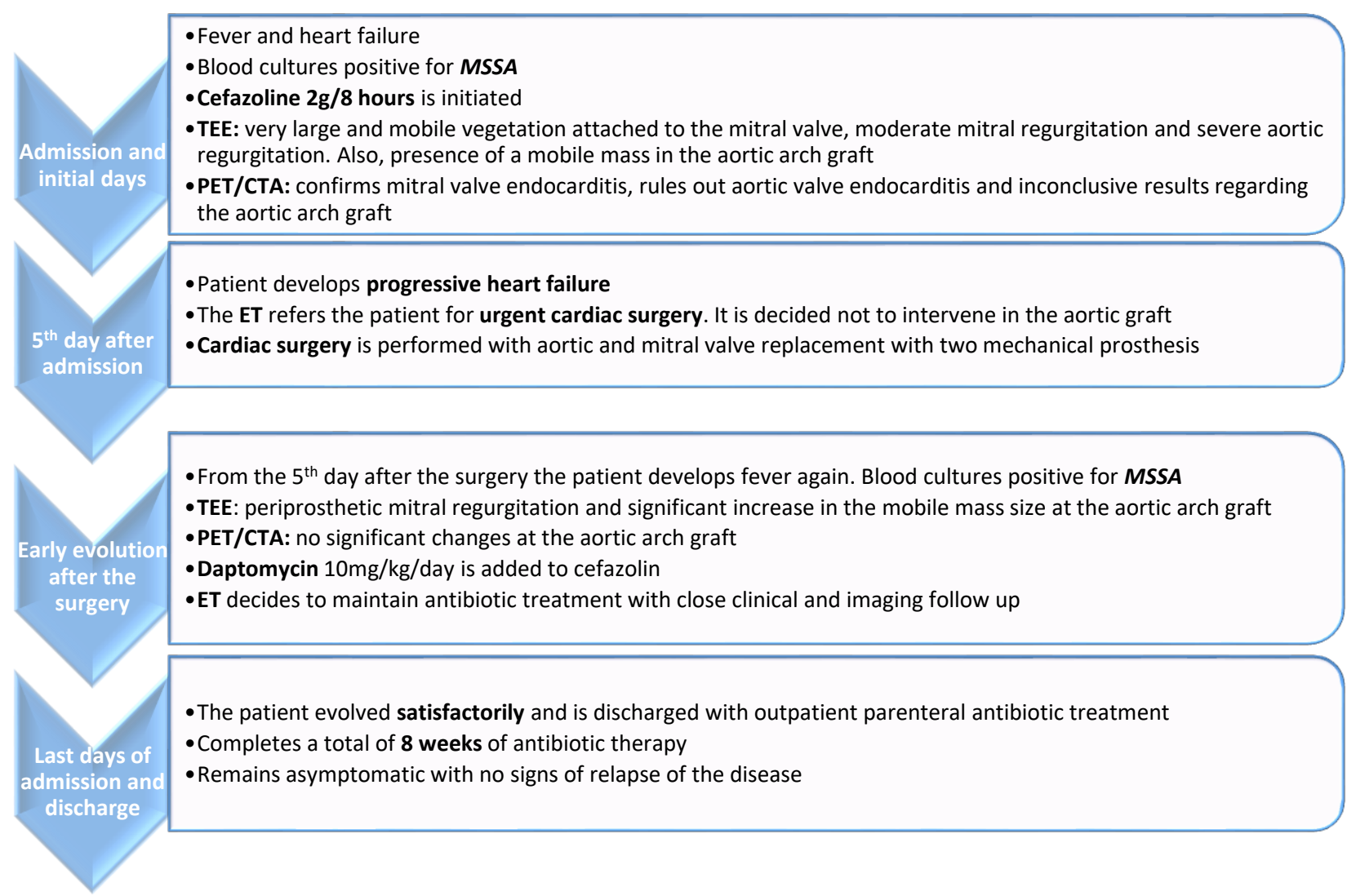

Fig. 4: Diagram of patient's evolution; ET: Endocarditis Team; MSSA: Methicillin-susceptible Staphylococcus aureus; PET/CTA: 18F-Fluorodeoxyglucose Positron Emission Tomography/Computed Tomography Angiography; TEE: Transesophageal Echocardiogram

The patient recovered satisfactorily in the following weeks with progressive decrease in size of the image of the aortic arch, stabilization of the mitral regurgitation and no evidence of infection of the aortic prosthetic valve. He had no more fever spikes and all blood cultures were negative. He was discharged home to continue with outpatient parenteral antibiotic therapy with cefazolin for 8 weeks. Five months after the initial 
diagnosis and two months after the end of the antibiotic treatment, the patient remains asymptomatic with no signs of relapse of the infection (Fig. 4).

\section{Discussion}

We present a case of a young patient with IE requiring complex medical and surgical decisionmaking that reinforces the role of the Endocarditis Team (Habib et al., 2015; Baddour et al., 2015). First, the patient had acute IE with a large vegetation and severe valvular and paravalvular lesions. It is known that patients with large vegetations have high embolic risk (Hubert et al., 2013) and in patients with severe valvular disease, urgent surgery is associated with a lower risk of embolization (Kang et al., 2012). However, in our case surgery was initially delayed to elucidate if the aortic arch graft was also infected.

The 18F-FDG-PET/CTA scan is a useful tool to rule-out or confirm the diagnosis of IE in possible prosthetic valve endocarditis or intracardiac device infection (Pizzi et al., 2015). Furthermore, infected aortic grafts may display focal and intense FDG uptake that may be associated with perigraft soft tissue reaction, so PET/CTA could also improve the diagnosis in some of these scenarios (Mitra et al., 2018; Erba et al., 2019). However, in some cases with suspected aortic graft infection the results of the PET/CTA can be inconclusive as in our case.

Aortic graft infection is an infrequent but serious complication with an infection rate of 1 to $5 \%$. The most frequent pathogens are Coagulase-negative staphylococci and Staphylococcus aureus (Wilson et al., 2016). If it is feasible, surgery is the optimal treatment although there is some evidence that in patients with high surgical risk who would require complex surgery as in our case, prolonged antibiotic therapy could be a reasonable alternative (Ramos et al., 2016; García-Arribas et al., 2021). Since we could not confirm the diagnosis of aortic graft infection and considering the extreme risk of an aortic arch surgery and the satisfactory evolution with the antibiotic treatment, we decided to maintain prolonged antibiotic treatment in our patient with good results. Although the evidence regarding the efficacy of a combination treatment with daptomycin and a $\beta$-lactam in MSSA bacteremia is scarce, this treatment has been used as salvage therapy with success in patients with persistent or complicated bacteremia, as in our case (Gudiol et al., 2017; Moise et al., 2013).

\section{Conclusion}

This case illustrates the importance of the Endocarditis Team when it comes to difficult decisions regarding diagnosis and management of a disease with challenging scientific evidence.

\section{Author's Contributions}

Pau Rello: Manuscript writing, literature search. Image analysis and postprocessing.

Teresa González-Alujas: Manuscript review and editing. Image analysis and postprocessing.

Laura Escolà-Verge: Manuscript writing, literature search. Manuscript review and editing.

Albert Roque: Manuscript writing, literature search. Manuscript review and editing. Image analysis and postprocessing.

Carlos Sureda, Toni Soriano-Colomé, Gerard Oristrell and Ignacio Ferreira-González: Manuscript review and editing.

Antonia Sambola: Manuscript review and editing. Literature search.

\section{Ethics}

This article is original and contains unpublished material. The authors declare no ethical issue. Informed consent was obtained from the patient.

\section{References}

Baddour, L. M., Wilson, W. R., Bayer, A. S., Fowler Jr, V. G., Tleyjeh, I. M., Rybak, M. J., ... \& American Heart Association Committee on Rheumatic Fever, Endocarditis and Kawasaki Disease of the Council on Cardiovascular Disease in the Young, Council on Clinical Cardiology, Council on Cardiovascular Surgery and Anesthesia and Stroke Council. (2015). Infective endocarditis in adults: diagnosis, antimicrobial therapy and management of complications: a scientific statement for healthcare professionals from the American Heart Association. Circulation, 132(15), 1435-1486. https://doi.org/10.1161/CIR.0000000000000296

Erba, P. A., Pizzi, M. N., Roque, A., Salaun, E., Lancellotti, P., Tornos, P., \& Habib, G. (2019). Multimodality imaging in infective endocarditis: an imaging team within the endocarditis team. Circulation, 140(21), 1753-1765.

https://doi.org/10.1161/CIRCULATIONAHA.119.0 40228

García-Arribas, D., Olmos, C., Vilacosta, I., PerezGarcía, C. N., Ferrera, C., Jerónimo, A., ... \& Maroto, L. (2021). Infective endocarditis in patients with aortic grafts. International Journal of Cardiology, 330, 148-157.

https://doi.org/10.1016/j.ijcard.2021.02.030

Gudiol, C., Cuervo, G., Shaw, E., Pujol, M., \& Carratalà, J. (2017). Pharmacotherapeutic options for treating Staphylococcus aureus bacteremia. Expert Opinion on Pharmacotherapy, 18(18), 1947-1963. https://doi.org/10.1080/14656566.2017.1403585 
Habib, G., Lancellotti, P., Antunes, M. J., Bongiorni, M. G., Casalta, J. P., Del Zotti, F., ... \& Zamorano, J. L. (2015). 2015 ESC guidelines for the management of infective endocarditis: the task force for the management of infective endocarditis of the European Society of Cardiology (ESC) endorsed by: European Association for CardioThoracic Surgery (EACTS), the European Association of Nuclear Medicine (EANM). European Heart Journal, 36(44), 3075-3128. https://doi.org/10.1093/eurheartj/ehv319

Hubert, S., Thuny, F., Resseguier, N., Giorgi, R., Tribouilloy, C., Le Dolley, Y., ... \& Habib, G. (2013). Prediction of symptomatic embolism in infective endocarditis: construction and validation of a risk calculator in a multicenter cohort. Journal of the American College of Cardiology, 62(15), 1384-1392. https://www.jacc.org/doi/full/10.1016/j.jacc.2013. 07.029

Kang, D. H., Kim, Y. J., Kim, S. H., Sun, B. J., Kim, D. H., Yun, S. C., ... \& Sohn, D. W. (2012). Early surgery versus conventional treatment for infective endocarditis. New England Journal of Medicine, 366(26), 2466-2473. https://doi.org/10.1056/NEJMoa1112843

Mitra, A., Pencharz, D., Davis, M., \& Wagner, T. (2018). Determining the diagnostic value of $18 \mathrm{~F}$ Fluorodeoxyglucose positron emission/computed tomography in detecting prosthetic aortic graft infection. Annals of Vascular Surgery, 53, 78-85. https://doi.org/10.1016/j.avsg.2018.04.028
Moise, P. A., Amodio-Groton, M., Rashid, M., Lamp, K. C., Hoffman-Roberts, H. L., Sakoulas, G., ... \& Rastogi, A. (2013). Multicenter evaluation of the clinical outcomes of daptomycin with and without concomitant $\beta$-lactams in patients with Staphylococcus aureus bacteremia and mild to moderate renal impairment. Antimicrobial Agents and Chemotherapy, 57(3), 1192-1200. https://doi.org/10.1128/AAC.02192-12

Pizzi, M. N., Roque, A., Fernández-Hidalgo, N., CuéllarCalabria, H., Ferreira-González, I., Gonzàlez-Alujas, M. T., ... \& Tornos, P. (2015). Improving the diagnosis of infective endocarditis in prosthetic valves and intracardiac devices with 18F-fluordeoxyglucose positron emission tomography/computed tomography angiography: initial results at an infective endocarditis referral center. Circulation, 132(12), 1113-1126. https://doi.org/10.1161/CIRCULATIONAHA.115.0 15316

Ramos, A., García-Montero, C., Moreno, A., Muñoz, P., Ruiz-Morales, J., Sánchez-Espín, G., .. \& G GarcíaPavía, P. (2016). Endocarditis in patients with ascending aortic prosthetic graft: a case series from a national multicentre registry. European Journal of Cardio-Thoracic Surgery, 50(6), 1149-1157. https://doi.org/10.1093/ejcts/ezw190

Wilson, W. R., Bower, T. C., Creager, M. A., AminHanjani, S., O'Gara, P. T., Lockhart, P. B., ... \& Baddour, L. M. (2016). Vascular graft infections, mycotic aneurysms and endovascular infections: a scientific statement from the American Heart Association. Circulation, 134(20), e412-e460. https://doi.org/10.1161/CIR.0000000000000457 